\title{
Threshold strategy to improve the images reconstructed by electrical impedance tomography
}

\author{
Xiaoyan Chen, Jing Zhang \\ College of Electronic Information and Automation, Tianjin University of Science and Technology, Tianjin, China \\ Email: cxywxr@tust.edu.cn, zhangjingmza@126.com
}

Received 2013

\begin{abstract}
Because of the illposedness of soft field, the quality of EIT images is not satisfied as expected. This paper puts forward a threshold strategy to decrease the artifacts in the reconstructed images by modifying the solutions of inverse problem. Threshold strategy is a kind of post processing method with merits of easy, direct and efficient. Reconstructed by Gauss-Newton algorithm, the simulation image's quality is improved evidently. We take two performance targets, image reconstruction error and correlation coefficient, to evaluate the improvement. The images and the data show that threshold strategy is effective and achievable.
\end{abstract}

Keywords: Electrical Impedance Tomography; Threshold Strategy; Reconstruction Algorithm; Image Evaluation

\section{INTRODUCTION}

Electrical impedance tomography (EIT) is an attractive imaging technique which aims to estimate the interior conductivity or resistivity distribution of an unknown object. In EIT, an array of electrodes are attached to the boundary of an object, safe alternating currents are injected through electrodes, and the resulting voltages are measured to reconstruct the distributions using specific algorithms. Compared to the conventional medical imaging techniques, such as $\mathrm{X}$-ray computerized tomography (CT) or magnetic resonance imaging (MRI), EIT has the advantages of low cost, no radiation and easy portability and suitable for clinical monitoring applications [1-3]. Despite its relatively poor spatial resolution, EIT, as a tool for imaging, has been studied for over 30 years [4-7].

With Maxwell's electromagnetic field theory [8], the physical model of the sensitive field for EIT system can be derived. Apart from the physical model in the region, the current injection and voltages measurements on $\partial \Omega$ are described by imposing appropriate boundary condi- tions. The complete electrode model (CEM) $[9,10]$ is one of the most sophisticated electrode models describing the relationship between the boundary voltages and applied currents [11]. Considering the conductive nature of the electrodes and the drop across the contact impedance $z_{l}$, the equations describing the CEM of EIT system can be described as follows.

$$
\left.\begin{array}{l}
\nabla \cdot(\sigma \nabla \mu)=0 \\
\sigma \frac{\partial u}{\partial \mathbf{n}}=0 \quad u \in \partial \Omega \backslash\left\{e_{1} \cup \cdots \cup e_{L}\right\} \\
\int_{e_{l}} \sigma \frac{\partial u}{\partial \mathbf{n}} d s=I_{l} \quad u \in \partial \Omega \quad l=1,2, \cdots, L \\
u+z_{l} \sigma \frac{\partial u}{\partial \mathbf{n}}=V_{l} \quad u \in \partial \Omega \quad l=1,2, \cdots, L \\
\sum_{l=1}^{L} V_{l}=0 \\
\sum_{l=1}^{L} I_{l}=0
\end{array}\right\}
$$

where $\mathbf{n}$ is the outward pointing normal vector to $\partial \Omega$, $\sigma$ is the conductivity, $u$ is the electric potential, $z_{l}$ is the effective contact impedance between the $l$ 'th electrodes and the body. $I_{l}$ and $V_{l}$ is the injected current and potential at each electrodes.

This paper puts forward a threshold strategy to decrease the artifacts in the reconstructed images. The method is described in Section 2. The reconstructed images are shown in Section 3. Image reconstruction error, correlation coefficient and the evaluations are introduced in Section 4. Experimentations results are completed in Section 5 .

\section{METHODOLOGY}

Image reconstruction in EIT is a nonlinear problem, which aims at approximating the interior conductivity distribution by injected electrical currents and measured resulting boundary voltages.

Difference EIT calculates a vector of conductivity change between the object and the reference background. 
Under the assumption that only small deviations from the reference conductivity exist, the inverse problem for EIT can be solved for linear reconstruction accurately and rapidly, Gauss-Newton algorithm, which has been widely used in EIT since the late 1980s [12-14] is taken to calculate image reconstruction matrix in our research.

The solutions in the reconstruction matrix reconstruct the images. The artifacts in the image are caused by some unsatisfied solutions. If a threshold is set to adjust the value within an appropriate range, the artifacts can be cut off sharply, spatial resolution should be improved. Suppose, there are $m$ pixels in a field refined by finite element method (FEM), $x_{i}$ is the inverse solution of pixel $i(i=1,2,3 \cdots m), P$ is the threshold factor, 0 or 1 , $p, q$ is the threshold values, which satisfied $\min \left(x_{i}\right) \leq q \leq 0 \leq p \leq \max \left(x_{i}\right)$. The conditions

$\sigma_{\text {target } 1} \leq \sigma_{\text {background }} \leq \sigma_{\text {target } 2}$ is under investigation. Then, the conductivity can by modified as

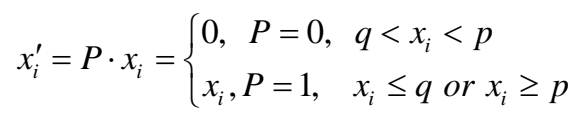

Make the conductivity closed to that of the background zero. Only the higher values over the threshold are kept their numerical solution. With this modification, the reconstruction matrix is refreshed and the artifacts of the images expected to be reduced.

\section{RECONSTRUCTION IMAGES}

The reconstructed image is refined into 12 layers and 576 elements by FEM. In simulation, we set to foreign targets in a homogenous circle. The conductivities are set to $\sigma_{\text {background }}=1, \sigma_{\text {target } 1}=0.5, \sigma_{\text {target } 2}=2$. The reconstruction images are shown in Figure 1. The trial-anderror method is adopted to decide the threshold. We got $q=-0.155, p=0.18$ for GN solutions.

In Figure 1, blue blocks are the lower conductivity targets and red blocks are the higher conductivity targets.

\section{EVALUATIONS}

We elaborate two targets to characterize the quality of a reconstructed image. Clearly, there are several criteria proposed to evaluate the EIT systems, including hardware and algorithms $[15,16]$. Most comments of the images are lack of objectivity. In this paper, relative errors and the correlated coefficients of images are adopted to evaluate the modification effects.

\subsection{Relative Errors (RE)}

The definition of relative errors is

$$
R E=\frac{\left\|\mathbf{x}^{*}-\mathbf{x}\right\|}{\left\|\mathbf{x}^{*}\right\|}
$$

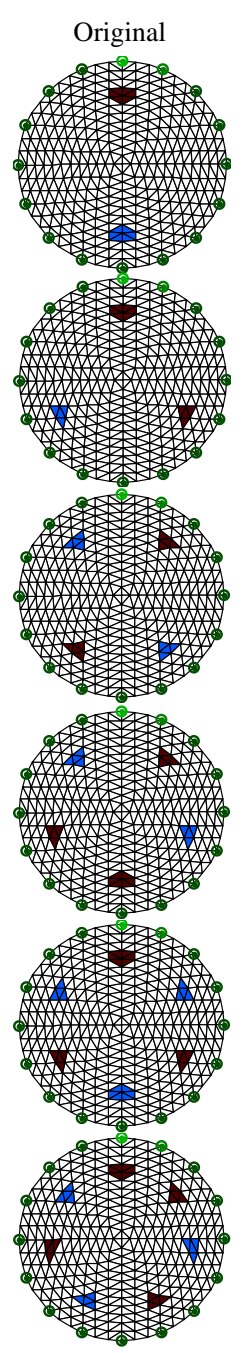

(a)

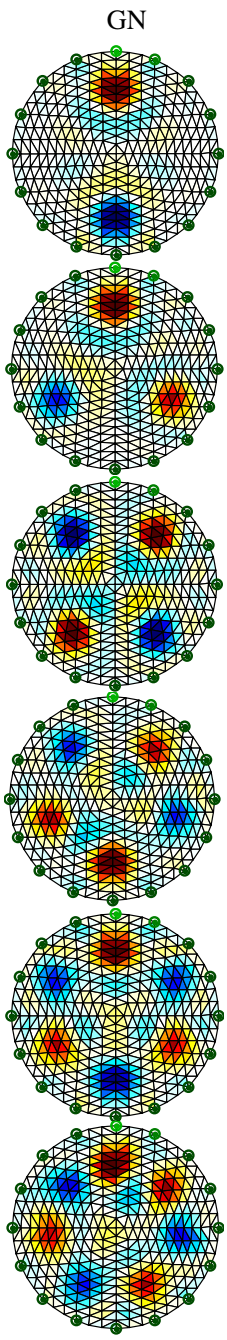

(b)

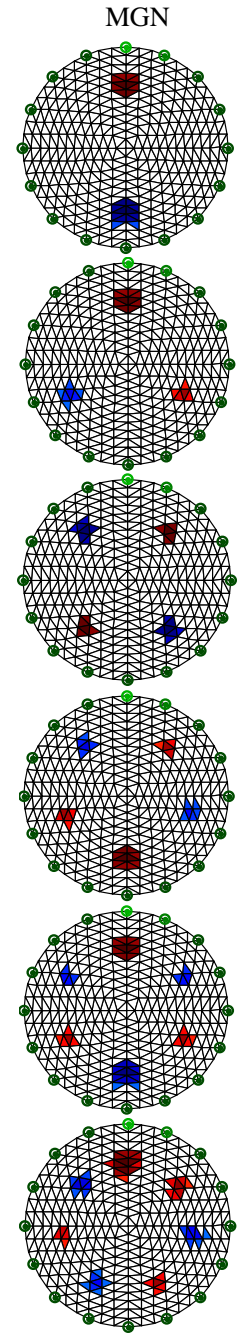

(c)
Figure 1. Simulation reconstructed images. (a) Original images; (b) Reconstructed images by GN; (c) Modified images by GN.

where, $\boldsymbol{x}^{*}$ is the normalized grey vector including all the pixels of the simulated image, while $\boldsymbol{x}$ presents that of the reconstructed image. The smaller RE means the higher quality of the reconstructed image.

\subsection{Correlated Coefficient (CC)}

Correlated coefficient (CC) is adopted to express the related degree of the reconstructed image to the simulated image, and calculated by

$$
C C=\frac{\sum_{i=1}^{N}\left(x_{i}-\bar{x}\right)\left(x_{i}^{*}-\bar{x}^{*}\right)}{\sqrt{\sum_{i=1}^{N}\left(x_{i}-\bar{x}\right)^{2} \sum_{i=1}^{N}\left(x_{i}^{*}-\bar{x}^{*}\right)^{2}}}
$$

where, $\boldsymbol{x}^{*}$ is the normalized grey vector including all the pixels of the simulated image, while $\boldsymbol{x}$ presents that of the reconstructed image. $\overline{\boldsymbol{x}}^{*}$ is the average value of 
$\boldsymbol{x}^{*}$, and $\overline{\boldsymbol{x}}$ is the average value of $\boldsymbol{x}$. If CC is closed to 1 , the reconstructed image is similar to the fact closely.

Figure 2 shows the relative errors and correlative coefficients of 2 - 7 subjects. The solid blue lines present data before modifying by threshold strategy and the dotted red lines present that after the modification. It is obvious that the dotted red lines are all lower than the solid blue lines in (a), which means that the threshold strategy improves the image quality, while the dotted red lines are almost above the solid blue lines in (b), which mean the modified images are more close to the true distribution.

From Figure 2, we can draw the conclusion that the average decrease of relative error by GN is about $18.27 \%$, and the average improvement of correlation coefficient by $\mathrm{GN}$ is about $11.71 \%$.

\section{EXPERIMENTATIONS AND CONCLUSIONS}

In the experiments, we collect the data by TJU-EIT system $[17,18]$ which has 16 metal electrodes attached around the boundary of a cylinder tank with $28 \mathrm{~cm}$ diameter. Salt water is background solution, and organic glass rods are the targets. As $\sigma_{\text {saltywater }} \geq \sigma_{\text {rods }}$, the threshold value is the same as Figure 1. Here, GN algorithm is accomplished. The images are shown in Figure $\mathbf{3}$ and the performance targets are compared in Figure 4.

From above figures, the images get clearer and the artifacts are decreased obviously. The RE is decreased to $47.69 \%$, CC is improved $11.26 \%$.

This study proposes a threshold strategy to decrease

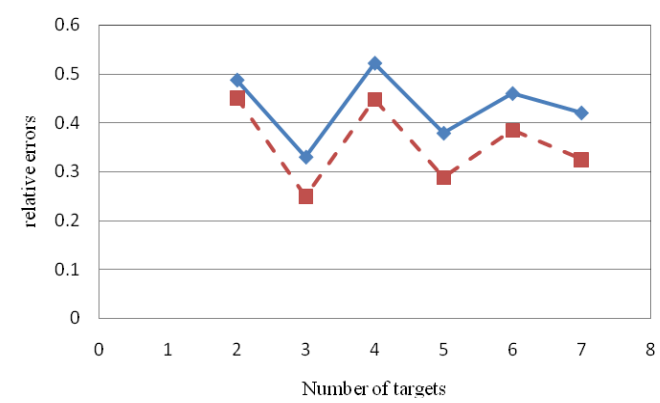

(a)

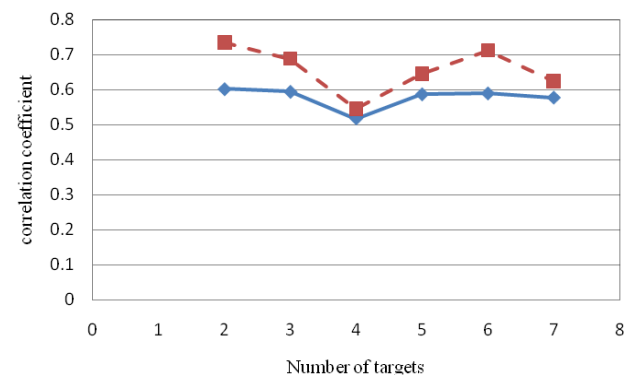

(b)

Figure 2. Relative errors and correlation coefficients.
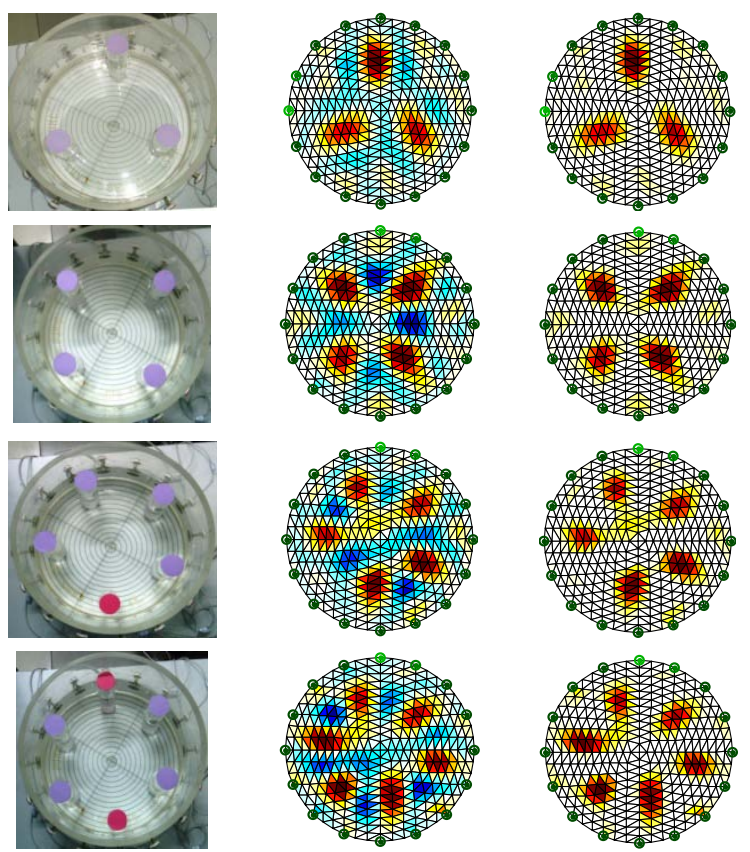

(b)

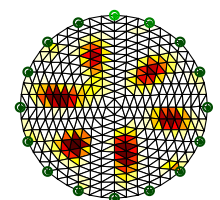

(c)

Figure 3. Experimental reconstructions. (a) Original images; (b) Images by GN directly; (c) Images by GN modified.

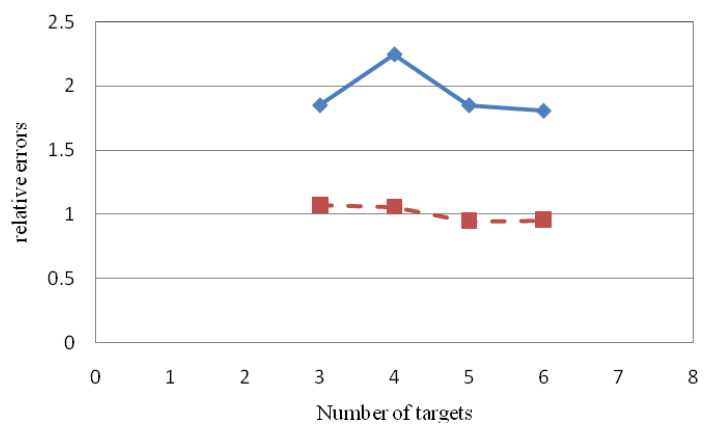

(a)

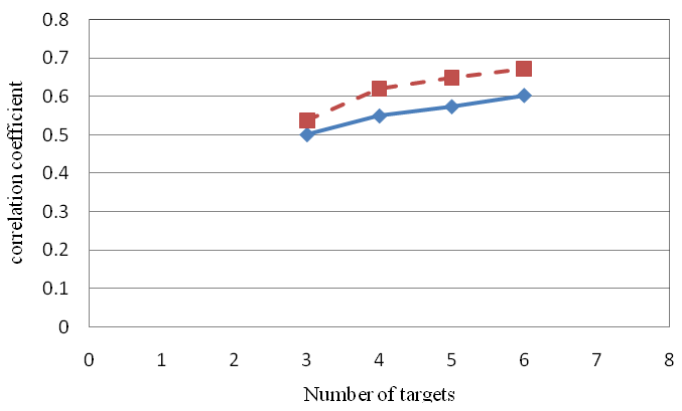

(b)

Figure 4. Relative error and correlation coefficient curves of the previous and post-processing by GN (a) The relative errors of the $3-6$ objects, (b) The correlation coefficients of the 3 - 6 objects.

the artifacts in the images. The images are reconstructed by Gauss-Newton algorithm. Through trial and error tests, the appropriate threshold conductivities are adopted to 
modify the reconstructed matrix, and the modified images are achieved and evaluated through the relative error and correlation coefficient, respectively. Simulation and experimental results show that the artifacts are reduced and the images are improved significantly, which prove that the proposed approach makes significant contribution in improving EIT images.

\section{ACKNOWLEDGEMENTS}

This work is supported by Natural Science Foundation of China (GN: 50937005) and the Natural Science Foundation of Tianjin Municipal Science and Technology Commission (GN: 08JCYBJC03500). The authors wish to express their special gratitude to Professor Chaoshi Ren, Chinese Academy of Medical Science and Peking Union Medical College Institute of Biomedical Engineering for his sincerest academic advises on this paper.

\section{REFERENCES}

[1] Brown, B.H., Primhak, R.A., Smallwood, R.H., Milnes, P., Narracott, A.J. and Jackson, M.J. (2002) Neonatal lungs-maturational changes in lung resisitivity spectra. Journal of Medical and Biological Engineering, 40, pp. 506-511. http://dx.doi.org/10.1007/BF02345447

[2] Kerrouche, N., McLeod, C.N. and Lionheart, W.R.B. (2001) Time series of EIT chest images using singular value decomposition and Fourier transform. Physiological Measurement, 22, 147-158. http://dx.doi.org/10.1088/0967-3334/22/1/318

[3] Kulkarni, R., Kao, T.J., Boverman, G., Isaacson, D., Saulnier, G.J. and Newell, J.C. (2009) A two-layered forward model of tissue for electrical impedance tomography. Physiological Measurement, 30, 19-34. http://dx.doi.org/10.1088/0967-3334/30/6/S02

[4] Newell, J.C., Gisser, D.G. and Isaacson, D. (1988) An electric current tomography. IEEE Transactions on Biomedical Engineering, 35, 828-833. http://dx.doi.org/10.1109/10.7289

[5] Morucci, J.P. and Marsili, P.M. (1996) Bioelectrical impedance techniques in medicine Part III: Impedance imaging. Critical Reviews in Biomedical Engineering, 24, 599-654.

[6] Hanke, M. and Bruhl, M. (2003) Recent progress in electrical impedance tomography. Inverse Problems, 19, 65-

\section{0. http://dx.doi.org/10.1088/0266-5611/19/6/055}

[7] Holder, D.S. (2004) Electrical impedance tomography methods: history and applications. Institute of Physics Pub, Bristol, 1-65.

[8] Ida, N. and Bastos, J.P.A. (1997) Electromagnetics and calculation of fields. 2nd Edition, Springer-Verlag Inc., New York. http://dx.doi.org/10.1007/978-1-4612-0661-3

[9] Polydorides, N. (2002) Image reconstruction algorithms for soft-field tomography. PhD Thesis, UMIST.

[10] Vauhkonen, P.J., Vauhkonen, M., Savolainen, T. and Kaipio, J.P. (1999) Three dimensional electrical impedance tomography based on the complete electrode model. IEEE Transactions on Biomedical Engineering, 46, 11501160. http://dx.doi.org/10.1109/10.784147

[11] Somersalo, E., Cheney, M. and Isaacson, D. (1992) Existence and uniqueness for electrode models for electric current computed tomography. SIAM Journal on Applied Mathematics, 52, 1023-1040.

[12] Dan, F. and Hagan, F.M.T. (1997) Gauss-Newton approximation to Bayesian learning. International Conference on Neural Networks, 3, 1930-1935.

[13] Bernhard, B., et al. (2003) Direct estimation of Cole parameters in multifrequency EIT using a regularized GaussNewton method. Physiological Measurement, 24, 437. http://dx.doi.org/10.1088/0967-3334/24/2/355

[14] Andy, A., John, H.A. and Richard, B. (2009) GREIT: A unified approach to 2D linear EIT reconstruction of lung images. Physiological Measurement, 30, 35-55. http://dx.doi.org/10.1088/0967-3334/30/6/S03

[15] Tanguay, L.F., Gagnon, H. and Guardo, R. (2007) Comparison of applied and induced current electrical impedance tomography. IEEE Transactions on Biomedical Engineering, 54, 1643-1649. http://dx.doi.org/10.1109/TBME.2007.892930

[16] Ryan, H., Alex, H. and Keith, D.P. (2004) Design and implementation of a high frequency electrical impedance tomography system. Physiological Measurement, 25, 379390. http://dx.doi.org/10.1088/0967-3334/25/1/041

[17] Chen, X.Y., Wang, H.X., et al. (2008) Lung ventilation imaging with prior information by Electrical Impedance Tomography. IEEE IMTC, 1531-1537.

[18] Chen, X.Y., Wang, H.X., et al. (2009) Lung ventilation functional monitoring based on Electrical Impedance Tomography. Transactions of Tianjin University, 15, 7-12. 